\title{
Amaranto, lípidos y su efecto en las dislipidemias
}

\author{
Amaranth, lipids and its effect in dyslipidemias \\ Gustavo Peña Serrano ${ }^{a}$, José Alberto Ariza Ortega ${ }^{b}$
}

\begin{abstract}
:
Amaranth grain (Amaranthus) is a pseudocereal with chemical composition and cooking similar to cereals like wheat, corn and rice, it is used to elaborate bread and its derivatives. It contains carbohydrates (75.3-76.8 g/100 g), proteins (12-19 g/100 g), lipids (6.1$8.1 \mathrm{~g} / 100 \mathrm{~g})$, water soluble vitamins $(6.11 \mathrm{~g} / 100 \mathrm{~g})$, lipid soluble vitamins $(63.7-129.3 \mathrm{mg} / 100 \mathrm{~g})$ and minerals $(3-3.1 \mathrm{~g} / 100 \mathrm{~g})$. Among its lipids predominate polyunsaturated fatty acids $(41.85 \%)$ and squalene $(52.2 \mathrm{mg} / \mathrm{g}$ of oil). Squalene is a terpene found in significant amounts in amaranth oil, furthermore, it has antioxidant capacity and it inhibits free radicals, next to fiber it could improve the lipid profile in dyslipidemias, however, its consumption it's not common. Therefore, this works objective was to analyze amaranth theorist element to know the grain and lipids consumption effect in dyslipidemia. This work was a documentary type investigation, in which a search was carried out in databases such as PubMed and Science Direct. As a result of this work, experimental animals' studies demonstrated that intake of amaranth grain with a fiber content $(3.5-5.0 \mathrm{~g} / 100 \mathrm{~g})$, can prevent cholesterol raise $73 \%(200 \mathrm{mg} / \mathrm{dL})$ during hypercholesterolemic diets intake, due to a fiber viscosity increase in intestinal mucosa, this prevents its absorption. On the other hand, in squalene enriched diets $(1 \mathrm{~g} / \mathrm{kg})$, and owing to the double bonds in its chemical structure, it neutralizes Reactive Oxygen Species (ROS) in LDL cholesterol $\left(22.5 \times 10^{5}-4 \times 10^{5}\right.$ Fluorescence Arbitrary Units) and increases HDL cholesterol (0.4-0.6 mM). So, the fiber and lipids content are efficient in dyslipidemia control or prevention.
\end{abstract}

Keywords:

Amaranthus, amaranth oil, squalene, dyslipidemia, cholesterol.

\section{Resumen:}

El grano de amaranto (Amaranthus) es un pseudocereal con una composición química y cocción similar a cereales como trigo, maíz y arroz, se utiliza para elaborar pan y derivados. Contiene carbohidratos (75.3-76.8 g/100 g), proteínas (12-19g/100g), lípidos (6.1$8.1 \mathrm{~g} / 100 \mathrm{~g})$, vitaminas hidrosolubles $(6.11 \mathrm{~g} / 100 \mathrm{~g})$, vitaminas liposolubles $(63.7-129.3 \mathrm{mg} / 100 \mathrm{~g})$ y minerales $(3-3.1 \mathrm{~g} / 100 \mathrm{~g})$. Entre sus lípidos, predominan los ácidos grasos poliinsaturados $(41.85 \%)$ y escualeno $(52.2 \mathrm{mg} / \mathrm{g}$ de aceite). El escualeno, es un terpeno que se encuentra en cantidades importantes en su aceite, tiene capacidad antioxidante e inhibe radicales libres, y junto con la fibra podría mejorar el perfil lipídico en las dislipidemias, sin embargo, su consumo no es habitual. Por lo anterior, el objetivo fue analizar los elementos teóricos del amaranto, para conocer el efecto del consumo del grano y sus lípidos sobre las dislipidemias. Este trabajo fue una investigación de tipo documental, en el que se realizó una búsqueda en bases de datos electrónicas como PubMed y Science Direct. Como resultado de esta investigación, se demostró que en animales de experimentación solo la ingesta del grano de amaranto con un contenido de fibra (3.5-5.0 g/100 g), evitó el incremento de colesterol total en $73 \%$ (200 mg/dL) durante la ingesta de dietas hipercolesterolémicas, debido a que la fibra incrementa su viscosidad en la mucosa intestinal, lo cual evita su absorción. Por otra parte, en dietas enriquecidas en escualeno $(1 \mathrm{~g} / \mathrm{kg})$, y debido a sus dobles enlaces en su estructura química, neutralizó especies reactivas de oxígeno en colesterol LDL (22.5 $\times 10^{5}-4 \times 10^{5}$ Unidades Arbitrarias de Fluorescencia) y aumentó el colesterol HDL (0.4-0.6 mM). Por lo tanto, el contenido de fibra y lípidos es eficaz para controlar o prevenir dislipidemias.

\section{Palabras Clave:}

Amaranthus, aceite de amaranto, escualeno, dislipidemia, colesterol.

Autor de Correspondencia, Universidad Autónoma del Estado de Hidalgo, https://orcid.org/0000-0001-8319-5242, Email: gustavopesser@gmail.com

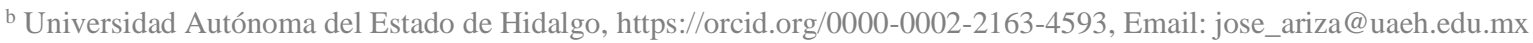




\section{Tabla 1}

Principales variedades de amaranto cultivadas a nivel mundial.

País Variedad cultivada

México

Amaranthus

hypochondriacus

Guatemala /

Zimbabue

Amaranthus cruentus

Perú

Amaranthus caudatus

India / USA

Amaranthus tricolor

Tanzania

Amaranthus hybridus

Kenia

Amaranthus dubios

\section{Introducción}

\section{Amaranto}

El amaranto es una planta frondosa con una altura (dependiendo de la especie) entre los $0.5-3 \mathrm{~m}$, con tallos gruesos y sus flores pueden ser de distintos colores (rojas,

anaranjadas, rosas, verdes o moradas) (1), se clasifica como un pseudocereal, por su apariencia y contenido de almidón, similar a la de los cereales, pero a diferencia de éstos, que pertenecen al grupo de las gramíneas y son monocotiledoneas (una hoja se forma del embrión), los pseudocereales como el amaranto, la quinoa o el trigo sarraceno, son dicotiledoneas (su embrión forma dos hojas) (2). Los pseudocereales también son ricos en fibra, proteína de alta calidad y minerales como calcio, hierro y zinc; vitaminas, polifenoles y fitoesteroles (3).

No existe mucha información acerca del cultivo de amaranto en el mundo, sin embargo, se sabe que las regiones principales donde se cultiva son en Sudamérica tropical, África, Centro y Sudeste asiático, y las regiones más cálidas de Norte América (1). En la Tabla 1 se encuentran las principales variedades de amaranto cultivadas $(4,5)$.

La importancia del grano de amaranto está creciendo, debido a que contiene compuestos químicos deseables en la alimentación, y su consumo genera beneficios a la salud y para proporcionar esos compuestos (Tabla 2), algunos industriales han propuesto alternativas de diversificación del grano, en productos como grano reventado, el cual se utiliza para la elaboración de dulces artesanales y otros productos como granola, alimentos integrales, frituras, panificados, pastas, galletas, aceites, papillas, concentrados proteicos, barras energéticas, entre otros (6).

\section{Tabla 2}

Composición química de la semilla de amaranto por cada $100 \mathrm{~g}$ de parte comestible

\begin{tabular}{ll}
\hline Característica & Contenido \\
\hline Proteína $(\mathrm{g})$ & $12-19$ \\
Carbohidratos $(\mathrm{g})$ & $75.3-76.8$ \\
Lípidos $(\mathrm{g})$ & $6.1-8.1$ \\
Cenizas $(\mathrm{g})$ & $3.0-3.3$ \\
Energía (kcal) & 391 \\
Vitaminas hidrosolubles $(\mathrm{mg})$ & 6.11 \\
Vitaminas liposolubles $(\mathrm{mg})$ & $63.7-129.3$ \\
\hline
\end{tabular}


Por otro lado, los beneficios a la salud que se le atribuyen al grano de amaranto se deben a las características de su composición química, que varía dependiendo de la especie y la variedad, así como de las condiciones climáticas (7), como se observa en la Tabla 2, puede llegar a superar el contenido proteico de cereales como el arroz ( $8.5 \%$ sobre el peso seco), el trigo ( $14 \%$ sobre el peso seco) o el maíz (10.3 \% sobre el peso seco), en el que se han detectado péptidos considerados como sustancias bioactivas potenciales (YESGSQ, GGEDE y NRPET, entre otros), ya que demuestran tener una actividad inhibitoria de la HMG-CoA reductasa (intermediaria en la vía del mevalonato en la síntesis de colesterol), dando como resultado un efecto hipocolesterolémico, así como un efecto antiinflamatorio, antihipertensivo y antimicrobiano $(8,9)$. Asimismo, una de las razones por las que se considera que la proteína del amaranto es de buena calidad, es porque además de contenerla en alta cantidad, en comparación con los cereales de mayor consumo, su proporción de aminoácidos esenciales, es muy similar a la de los alimentos de origen animal (10), ya que contiene leucina $(0.86 \mathrm{~g} / 100 \mathrm{~g})$, lisina $(0.83 \mathrm{~g} / 100 \mathrm{~g}) \mathrm{y}$ fenilalanina $(0.61 \mathrm{~g} / 100 \mathrm{~g})(11)$, además, el grano de amaranto contiene una cantidad muy baja de prolaminas (1.4\% del nitrógeno total) en comparación con las leguminosas $(15 \%)$, maíz, sorgo y otros cereales (alrededor del $45 \%$ ) $(12,13)$, por lo que puede ser una opción alimentaria para personas con enfermedad celíaca (7).

En la tabla 2 se observa que la semilla de amaranto contiene $71.8 \mathrm{~g} / 100 \mathrm{~g}$ de carbohidratos, donde el principal
Por otro lado, por cada $100 \mathrm{~g}$ de parte comestible, aporta de 3.5-5.0 g de fibra, cuya fracción soluble $(7-76 \mathrm{~g} / \mathrm{kg}$ ) está compuesta por pectina, ácido urónico, y biopolímeros de glucosa, arabinosa, galactosa, manosa y xilosa, y fibra insoluble $(72-194 \mathrm{~g} / \mathrm{kg})$. Este constituyente acelera la secreción de ácidos biliares y colesterol, que luego se une a la fibra y es eliminado con las heces, lo que evita que puedan ser reabsorbidos, contribuyendo así al control lipídico (14,17-20).

Debido a su alto contenido energético (371 kcal/100 g) (21), y su composición química similar a los cereales más consumidos (Trigo 352 kcal, Maíz 356 kcal, Arroz 358 kcal por cada $100 \mathrm{~g}$ ) (22), el amaranto podría sustituir a estos cereales en la dieta de poblaciones donde existe desnutrición, es una planta resistente al estrés ambiental y puede adaptarse a diferentes climas, lo cual facilita su cultivo (3).

Por otro lado, comparado con los cereales (trigo, arroz y maíz), el amaranto por cada 100 gramos, contiene niveles más elevados de fósforo (530 mg), calcio (130- $164 \mathrm{mg})$, potasio $(800 \mathrm{mg})$, magnesio $(248 \mathrm{mg})$ y hierro $(7.61 \mathrm{mg})$, también zinc y manganeso, las cantidades varían dependiendo de la especie de amaranto $(23,24)$.

Por último, por cada $100 \mathrm{~g}$ de parte comestible, el grano de amaranto contiene $4.2 \mathrm{mg}$ de vitamina $\mathrm{C}$, y vitaminas del complejo B, como B1 (0.12 mg), B2 (0.20 mg), B3 (0.92 $\mathrm{mg}$ ), B6 (0.59 mg), B9 (0.08 mg), cabe resaltar la importancia de su contenido de vitamina $E$, específicamente tocoferoles y tocotrienoles (63.7-129.3 $\mathrm{mg} / \mathrm{kg}$ ), debido a que demuestran tener actividad

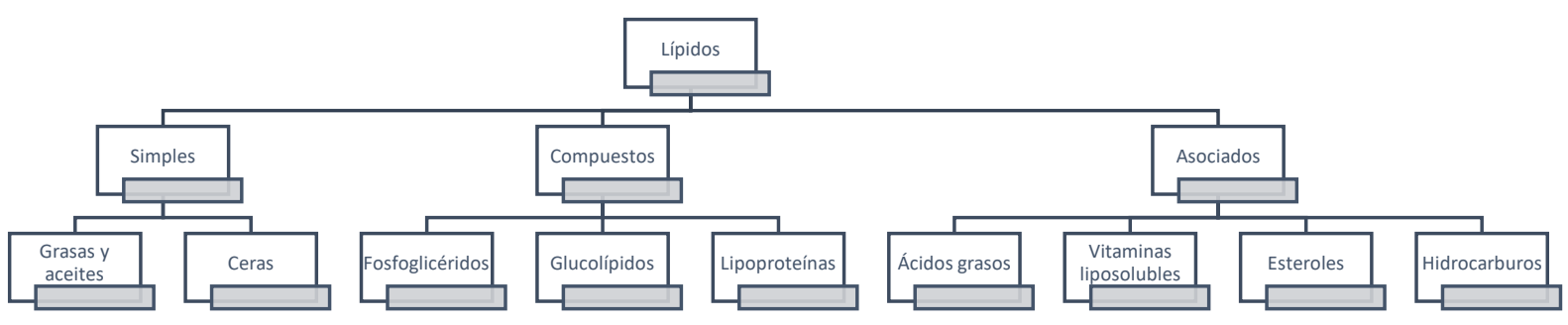

Figura 1. Clasificación de lípidos según su estructura molecular.

componente es el almidón, el cual equivale al $65-75 \%$ de la semilla de amaranto, el $1 \%$ es inositol, $0.05-0.67 \%$ de glucosa, fructuosa y otros monosacáridos, 0.27-2.3\% son disacáridos como la rafinosa, $0.4-2.00 \%$ sacarosa, $0.02-0.36 \%$ maltosa y $0.02-0.29 \%$ estaquiosa (14-16). antioxidante que resulta beneficiosa en enfermedades como la ateroesclerosis hipercolesterolémica (14,24-26), ya que, por acción de los radicales libres, las LDL (lipoproteínas de baja densidad, por sus siglas en inglés) pueden llegar a oxidarse, lo que modifica su actividad y es 
causa del desarrollo de ateroesclerosis, en este caso, los antioxidantes desempeñan un papel preventivo ante la oxidación de las LDL (27).

El grano de amaranto contiene entre $6.1-8.1 \mathrm{~g} / 100 \mathrm{~g}$ de lípidos, cuya composición es $30.51 \%$ ácidos grasos saturados, $27.46 \%$ monoinsaturados y $41.85 \%$ poliinsaturados (28). Su contenido de lípidos (7.2\% sobre el peso seco) es superior al del arroz (2.1\% sobre el peso seco), trigo ( $2.1 \%$ sobre el peso seco) y maíz ( $4.5 \%$ sobre el peso seco) (23). Por lo tanto, debido a su contenido mayoritario de ácidos grasos insaturados, puede reducir el riesgo de enfermedad cardiovascular (29).

\section{Lípidos}

Los lípidos son moléculas cuya principal característica es ser insolubles en agua y solubles en compuestos orgánicos, y se pueden clasificar en tres grupos principales con base en su estructura molecular $(12,13)$, como se muestra en la Figura 1.

Los lípidos simples son el grupo al que pertenecen las grasas, los aceites y las ceras, están formados por ésteres de ácidos grasos y alcoholes, como es el caso de los triglicéridos (Figura 2A), son los más abundantes y los más utilizados en la industria alimentaria. Por otro lado, los lípidos compuestos están formados por un lípido simple y una molécula no lipídica, que puede ser una proteína (lipoproteínas), fosfolípido o un carbohidrato (glucolípidos) como el $\beta-\delta$ galactosilceramida, que es un cerebrósido (Figura 2B). Por último, a los lípidos asociados pertenecen todos aquellos que, debido a sus características, no pueden ser clasificados como simples o compuestos, como los ácidos grasos, vitaminas liposolubles, esteroles, como el colesterol (Figura 2C), e hidrocarburos (20). A continuación, se muestran algunos ejemplos de lípidos de acuerdo a sus estructuras moleculares (Figura 2).

En general, los ácidos grasos son los más conocidos, y algunos de ellos son esenciales. Los ácidos grasos son moléculas que tienen en su estructura química una cadena hidrocarbonada no polar, que puede estar saturada o insaturada, y contiene un grupo carboxilo al final, y en menor cantidad fósforo y nitrógeno $(20,30)$, tales ácidos grasos se pueden encontrar, junto con fosfolípidos y constituyentes insaponificables, formando parte de la composición de los aceites vegetales (31) en forma de triglicérido (Figura 2A).
A B

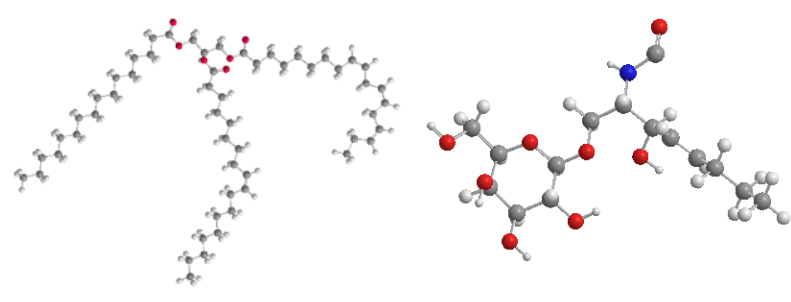

C

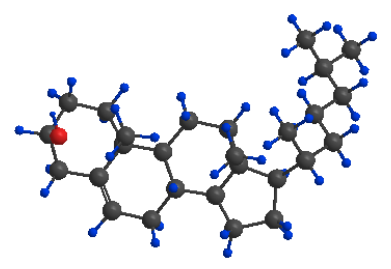

Figura 2. Ejemplos de estructuras químicas de los lípidos. Nota: Estructuras químicas de lípidos, donde $A$ : Triglicérido, B: $\beta-\delta$ Galactosilceramida y C: Colesterol. Estructura de bola y palo, realizada con Chem 3D versión 17.0.0.206 (Perkin Elmer Informatics, Inc. 1998 - 2017).

Los lípidos, se obtienen por medio de un prensado mecánico (aceite extra virgen) o por medio de una extracción química con disolventes orgánicos, como el hexano. Los rendimientos máximos con métodos mecánicos son del $80 \%$ y con la extracción con disolvente $98 \%$. Sin embargo, el uso del disolvente está prohibido por ser contaminante, y para eliminar el disolvente del aceite crudo, debe pasar por un proceso de refinamiento, donde se desechan sustancias indeseables que afectan las características organolépticas. No obstante, en las etapas de refinación (blanqueo) hay una disminución de tocoferoles y esteroles, como el escualeno. (31-33).

\section{Escualeno}

El escualeno, es un terpeno poliinsaturado con seis unidades de isopreno (Figura 3), es un precursor bioquímico del colesterol y otros esteroles, y puede encontrarse de forma endógena y exógena. En la forma endógena sintetizado por las células del hígado y la piel, en el caso de los humanos, donde se transporta en la piel por las VLDL (lipoproteínas de muy baja densidad, por sus siglas en inglés), y LDL (lipoproteínas de baja densidad, por sus siglas en inglés), es secretado por las glándulas sebáceas, cuya producción de cebo se compone por aproximadamente un 12-20\% por escualeno, el resto por glicéridos, colesterol, ésteres de colesterol y ácidos grasos libres. El escualeno producido por las glándulas sebáceas no es transformado a colesterol $(34,35)$. Su 
concentración en los lípidos de la piel es aproximadamente de $500 \mu \mathrm{g} / \mathrm{g}$, y en el tejido adiposo de $300 \mu \mathrm{g} / \mathrm{g}$, mientras que en órganos como el hígado y el intestino delgado se encuentran en concentraciones de 75 y $42 \mu \mathrm{g} / \mathrm{g}$ respectivamente $(32,36)$. Por otro lado, puede encontrarse de forma exógena en alimentos 0 suplementos, como fuente de origen animal como en el aceite de hígado de tiburón (50-81 \% de los lípidos totales) (37), el cual es acumulado en el hígado de las especies de aguas marinas, y debido a su baja densidad, cumple la función de proporcionar flotabilidad en conjunto con otros lípidos como triglicéridos, diacilglicéridos y ceras (38). También se encuentra en plantas, donde forma parte del centro de la bicapa lipídica celular, y mantiene el gradiente electroquímico para permitir el paso de los iones a través de la membrana (39). El escualeno se encuentra en cantidades importantes en el aceite oliva $(7 \mathrm{mg} / \mathrm{g}$ de aceite) (40), nuez (28.3 mg/g de aceite) y amaranto (52.2 $\mathrm{mg} / \mathrm{g}$ de aceite) (41). El escualeno forma parte de los antioxidantes, los cuales pueden clasificarse como endógenos (producidos por las células del organismo) o exógenos (obtenidos de los alimentos o suplementos), aunado a ello, es importante mencionar que el consumo de antioxidantes exógenos retarda el uso de los antioxidantes endógenos por parte de la célula, lo cual mantiene intacto su potencial antioxidante $(42,43)$. Por esto, se podrían realizar tratamientos dietéticos que incluyan alimentos con escualeno, más realizar ejercicio físico de intensidad moderada, para ejercer un efecto protector sobre las concentraciones de colesterol LDL y HDL (lipoproteínas de baja y alta densidad respectivamente, por sus siglas en inglés) y prevenir enfermedades como las dislipidemias (44).

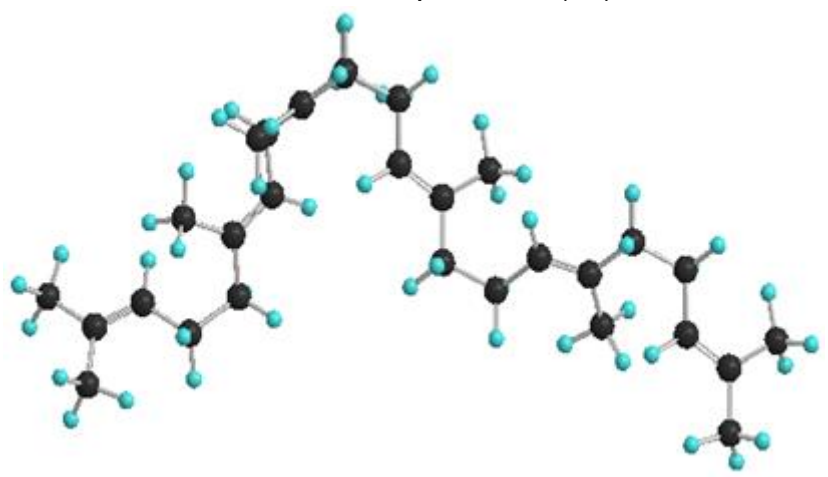

Figura 3. Estructura química del escualeno. Estructura de bola y palo realizada con Chem 3D versión 17.0.0.206 (Perkin Elmer Informatics, Inc. 1998 - 2017).

\section{Dislipidemias}

La dislipidemia es una enfermedad que se caracteriza por un desequilibrio en las concentraciones plasmáticas de lípidos, y que, en conjunto con otros padecimientos, como la obesidad, la hipertensión, resistencia a la insulina e hígado graso no alcohólico; conforma el síndrome metabólico, el cual representa un problema de salud pública que se relaciona directamente con el estilo de vida y la alimentación de las personas (45).

La concentración sérica de los lípidos en la sangre influye en las condiciones de salud del paciente (Tabla 3) (46), y si se encuentran presentes otros factores de riesgo como obesidad, diabetes, hipertensión arterial o tabaquismo, hay un deterioro progresivo de la salud, lo cual origina enfermedades crónicas que si no son atendidas a tiempo pueden causar la muerte $(47,48)$.

El tratamiento inicial de una dislipidemia se basa en el manejo de las posibles causas, como son el estilo de vida, consumo de carbohidratos, sedentarismo, diabetes, etc., y de acuerdo con la Guía de Práctica Clínica para el diagnóstico y tratamiento de las dislipidemias, se recomienda el consumo de una dieta saludable como base para la prevención, por lo que es necesario proporcionar un tratamiento nutricional efectivo, reduciendo el consumo de grasas saturadas y grasas trans, y sustituirlas por grasas insaturadas (omega 3 y 6). Además, es recomendable la disminución de peso para mejorar el control de la tensión arterial y la prevención de la diabetes (49). Además, se pueden adicionar a la dieta alimentos que contengan compuestos bioactivos, como lo es el grano de amaranto. Por lo tanto, el objetivo de este estudio fue analizar los elementos teóricos y experimentales del amaranto, para conocer el efecto del consumo del grano y sus lípidos en las dislipidemias. 


\section{Tabla 3}

Clasificación diagnóstica de las dislipidemias.

Dislipidemia

Criterios diagnósticos $(\mathrm{mg} / \mathrm{dL})$

Hipercolesterolemia

Hipercolesterolemia leve

Hipercolesterolemia moderada

Hipercolesterolemia severa

Hipertrigliceridemia

Dislipidemia mixta o combinada

Hipoalfalipoproteinemia
CT >200, TG<200 y C-LDL $\geq 130$

CT 200-239

CT $240-300$

$\mathrm{CT}>300$

TG $>300$, CT $<200$ y C-LDL $<130$

CT >200, TG >200 y C-LDL $\geq 130$

Nota: Clasificación de las dislipidemias donde CT = Colesterol Total, TG = Triglicéridos, C-LDL = Colesterol asociado a Lipoproteínas de baja densidad, C-HDL = Colesterol asociado a Lipoproteínas de alta densidad.

\section{Materiales y métodos}

Este trabajo fue una investigación de tipo documental, donde se realizó una búsqueda en bases de datos electrónicas como PubMed y Science Direct, desde el año 2015 hasta la fecha, tomando en cuenta estudios

realizados en animales de experimentación y humanos, usando palabras clave como: aceite de amaranto, amaranthus, colesterol, dislipidemia y escualeno. Sin embargo, la cantidad de artículos fue muy limitada (diez artículos), por lo que se decidió incluir artículos de años anteriores, de los cuales se seleccionaron siete. siendo un total de nueve los utilizados para esta revisión, dos con fecha posterior a 2015.

\section{Resultados}

En la Tabla 4 se presentan nueve estudios que usaron amaranto en el control de la dislipidemia, cinco usaron el grano de amaranto y el aceite en una dieta (50-54), de los cuales cuatro fueron en animales, y uno en humanos. En general, el grano y aceite de amaranto incrementaron las concentraciones de HDL y disminuyeron el colesterol total, LDL y VLDL, y en humanos con obesidad, se ha demostrado que la ingesta de aceite de amaranto, en conjunto con un plan de reducción de peso, mejora el perfil lipídico, así como parámetros antropométricos (peso corporal, índice de masa corporal, circunferencia abdominal, circunferencias de cintura y cadera, masa grasa, masa magra y masa grasa visceral).

Por otro lado, estudios en animales (52,55-58), usaron otros compuestos del amaranto (aislados proteicos de harina de amaranto y escualeno), directo o mezclado con alimentos, demostrando que su consumo influye en el metabolismo del colesterol, reportando alteraciones en la excreción de colesterol y ácidos biliares en materia fecal, así como disminución de las especies reactivas de oxígeno.

\section{Discusión}

De acuerdo con la ENSANUT MC (2016), donde se reporta que al $44.5 \%$ de los adultos entrevistados alguna vez les han medido los niveles de colesterol, de los cuales, el $28 \%$ (27.4\% hombres y $28.4 \%$ mujeres) dijeron haber recibido un diagnóstico previo de hipercolesterolemia. Siendo adultos de 50-79 años quienes tienen la mayor prevalencia. Valores que son menores a los presentados por la ENSANUT (2018), que indica que en los últimos años ha habido un aumento en el colesterol y triglicéridos 
de 7 y $6 \%$ en mujeres y hombres, respectivamente, siendo las mujeres quienes presentan valores más elevados de colesterol (>200 $\mathrm{mg} / \mathrm{dL})$ y triglicéridos (>150 $\mathrm{mg} / \mathrm{dL})(21 \%$ de las mujeres de 20 años o más) comparado con los hombres (17.7\%). Estos valores de colesterol y triglicéridos son factores de riesgo para el desarrollo de síndrome metabólico y enfermedad cardiovascular, una de las principales causas de morbilidad y mortalidad en el mundo (OMS, 2017) y representa el $80 \%$ de la mortalidad en nuestro país (OMS, 2013) (59-62).

La incidencia de estos padecimientos puede deberse a la presencia de trastornos enzimáticos y metabólicos que afectan la síntesis y eliminación de proteínas, como en el caso de la hipercolesterolemia familiar a hipercolesterolemia familiar combinada, así como la disbetalipoproteinemia e hipertrigliceridemia familiar, enfermedades en las que el colesterol LDL se encuentra elevado (desde una temprana edad), debido a la mutación del gen de la apolipoproteína $\beta$ (dislipidemia primaria). Otro factor para la incidencia de este padecimiento puede ser la presencia de alteración lipídica por la existencia o secuela de una enfermedad, como la diabetes mellitus, síndrome nefrótico, hipotiroidismo u obesidad. También el consumo de fármacos y exceso de alcohol o mala alimentación afectan al metabolismo lipídico (dislipidemia secundaria) (63).

Por lo anterior, la prevención y el tratamiento son importantes, una opción es el incremento en el consumo de alimentos con compuestos bioactivos, como el grano de amaranto, que contiene lípidos con dobles enlaces y fibra, donde se ha reportado que los ácidos grasos monoinsaturados y poliinsaturados presentes en el aceite de amaranto, ya que se ha indicado que los ácidos grasos monoinsaturados tienen un efecto antiinflamatorio que evita la actividad del inflamasoma NLRP3. Además, las dietas con alto contenido de ácidos grasos monoinsaturados, o el reemplazo de los ácidos grasos saturados por monoinsaturados, tiene un efecto positivo a la salud, mejorando la distribución de grasa abdominal, la sensibilidad a la insulina y el estrés oxidativo postprandial en pacientes con síndrome metabólico (64).

También se ha demostrado que el escualeno (SQ) tiene actividad antioxidante, reacción que ocurre cuando el escualeno libera un electrón al unirse al superóxido (O2- ) y formando hidroperóxido de escualeno (HOO-SQ) (65). La reacción es posible gracias a los dobles enlaces presentes en la estructura del escualeno, que lo convierten en un fuerte antioxidante y extremadamente reactivo al entrar en su forma oxidada, donde se une al hidrógeno del agua y libera tres iones de oxígeno, desarrollando su forma saturada. Debido a esto el oxígeno es capaz de alcanzar las células, intensificar el metabolismo celular y mejorar la función de órganos como el hígado y el riñón (44). Por demás, existen estudios que muestran el mecanismo molecular del escualeno, donde se ha reportados que es un regulador de los receptores $X$ del hígado, que se encargan de la expresión de genes que son clave en el transporte reverso del colesterol, sin inducir la lipogénesis en hepatocitos, lo que reduce el colesterol celular, permitiendo la salida de colesterol y evitando su acumulación in vitro e in vivo, así como del metabolismo de lípidos, carbohidratos y la fisiología de los macrófagos, inhibiendo así respuestas inflamatorias $(66,67)$.

Por otro lado, el contenido de fibra soluble del grano de amaranto, que incluyen a $\beta$-glucanos, fructanos (inulina, fructooligosacáridos), goma, pectina, mucilagos y fibras no viscosas como la hemicelulosa (68), dichas fibras son resistentes a la hidrólisis por las enzimas en humanos y son fermentadas por la microbiota intestinal en ácidos grasos de cadena corta, como acetato, propionato y butirato (69), donde se ha señalado al propionato como responsable del efecto hipocolesterolémico, ya que se relaciona con una disminución de la HMG-Co A sintasa y la HMG-Co A reductasa, enzimas mediadoras en la síntesis de colesterol (70-72). Por otra parte, estudios han demostrado que la ingesta de fibra incrementa la viscosidad, y esto limita la accesibilidad del colesterol a la superficie de la mucosa intestinal, evitando su absorción (73), esto reduce los niveles de colesterol plasmático $(74,75)$, además, la fibra también puede formar enlaces hidrofóbicos con las sales biliares, propiedad que depende de la cantidad y tipo de fibra, así como del tamaño de la partícula y su distribución. Lo que evita la formación de micelas y la reabsorción de los ácidos biliares, aumentando su excreción fecal, para compensar esta pérdida, se promueve la síntesis de ácidos biliares, resultando en una disminución del colesterol en plasma (73,76-79). Dicha unión puede ocurrir también entre la fibra soluble y la lipasa pancreática, por medio de fuerzas hidrofóbicas, electroestáticas, encapsulación o absorción, lo que genera un fuerte efecto inhibitorio (80). 
Tabla 4

Efecto del amaranto y el escualeno en el perfil lipídico.

\begin{tabular}{|c|c|c|c|c|c|}
\hline $\begin{array}{l}\text { Animal de } \\
\text { experimenta } \\
\text { ción }\end{array}$ & $\begin{array}{l}\text { Dietas, alimento y } \\
\text { concentración. }\end{array}$ & $\begin{array}{l}\text { Parámetros a } \\
\text { medir }\end{array}$ & $\begin{array}{l}\text { Tiempo de } \\
\text { intervención }\end{array}$ & Resultados & Referencia \\
\hline Gallinas & $\begin{array}{l}\text { Grano de amaranto: entero, } \\
\text { molido y reventado ( } 200 \\
\mathrm{~g} / \mathrm{kg}) \text { y aceite de amaranto } \\
(50 \mathrm{~g} / \mathrm{kg})\end{array}$ & Colesterol & 23 días & $\begin{array}{l}\text { El aceite de amaranto incrementó ligeramente el } \\
\text { colesterol } \mathrm{HDL} \text { en } 6 \%(1.97-2.09 \mathrm{mmol} / \mathrm{L}) \text {. } \\
\text { El amaranto reventado disminuyó los niveles de LDL en } \\
70 \%(1.23-0.52 \mathrm{mmol} / \mathrm{L}) \text {. }\end{array}$ & $\begin{array}{l}\text { Qureshi et } \\
\text { al. (1996) }\end{array}$ \\
\hline Conejos & $\begin{array}{l}\text { Grano de amaranto } \\
\text { reventado (5 g/día) (G2), } \\
\text { estándar (G3), } \\
\text { hipercolesterolémica con } \\
\text { amaranto (G4), } \\
\text { hipercolesterolémica (G5). }\end{array}$ & $\begin{array}{l}\text { Peso corporal, } \\
\text { perfil lipídico, } \\
\text { colesterol y ácidos } \\
\text { biliares } \\
\text { excretados, } \\
\text { función endotelial. }\end{array}$ & 21 días & $\begin{array}{l}\text { Aumento de colesterol total y LDL en todas las dietas } \\
\text { (hasta } 750 \mathrm{mg} / \mathrm{dL} \text { ), pero fueron menores en } 73 \% \text { ( } 200 \\
\mathrm{mg} / \mathrm{dL} \text { ) con las dietas que contienen amaranto (G2 y G4). } \\
\text { Incremento de colesterol fecal (G2) en } 167 \% \text { (310-830 } \\
\mathrm{mg} / \mathrm{kg} \text { ) y (G3) del } 77 \% \text { ( } 310-550 \mathrm{mg} / \mathrm{kg}) \\
\text { Capacidad de relajación endotelial disminuida en dietas } \\
\text { sin amaranto. }\end{array}$ & $\begin{array}{l}\text { Caselato et } \\
\text { al. (2014) }\end{array}$ \\
\hline Hámsteres & $\begin{array}{l}\text { Dietas hipercolesterolémicas } \\
\text { con grano de amaranto ( } 20 \\
\% \text { y } 1.4 \% \text { de aceite de } \\
\text { amaranto) y aceite de } \\
\text { amaranto }(5 \%) .\end{array}$ & Colesterol & 4 semanas & $\begin{array}{l}\text { El aceite de amaranto }(5 \%) \text { disminuye el colesterol total } \\
(15 \%) \text { y las LDL }(22 \%) \text {. } \\
\text { El grano de amaranto disminuye el colesterol LDL e } \\
\text { incrementa el colesterol HDL. } \\
\text { El grano de amaranto y el aceite disminuyeron las VLDL } \\
(21-50 \%) \text { y aumentaron la excreción fecal de colesterol y } \\
\text { ácidos biliares. }\end{array}$ & $\begin{array}{l}\text { Berger et al. } \\
(2003)\end{array}$ \\
\hline Ratas & $\begin{array}{l}\text { Dieta con } 1 \% \text { colesterol y } \\
\text { grano de amaranto ( } 300 \\
\mathrm{~g} / \mathrm{kg}) \text { o aceite de amaranto } \\
(90 \mathrm{~g} / \mathrm{kg}) \text {. } \\
\text { Dieta de colesterol y } \\
\text { escualeno de amaranto o de } \\
\text { tiburón inyectado ( } 200 \\
\mathrm{mg} / \mathrm{kg}) \text {. }\end{array}$ & Perfil lipídico & $\begin{array}{l}4 \text { semanas } \\
7 \text { días } \\
\text { (inyección) }\end{array}$ & $\begin{array}{l}\text { El grano de amaranto disminuyó triglicéridos ( } 5.64-2.98 \\
\mathrm{mmol} / \mathrm{L}) \text { y colesterol ( } 8.4-6.63 \mathrm{mmol} / \mathrm{L}) \text { séricos y } \\
\text { hepáticos ( } 46.7-32.8 \mathrm{mmol} / \mathrm{g} \text { y } 71.6-57.6 \mathrm{mmol} / \mathrm{g} \\
\text { respectivamente). } \\
\text { El aceite disminuyó el colesterol sérico y hepático (8.4- } \\
6.10 \mathrm{mmol} / \mathrm{L} \text { y } 71.6-61.9 \mathrm{mmol} / \mathrm{g} \text { respectivamente), así } \\
\text { como la concentración de triglicéridos séricos y hepáticos } \\
\text { (5.64-2.38 mmol/L y } 46.7-36.7 \mathrm{mmol} / \mathrm{g} \text { respectivamente). } \\
\text { También mostró un aumento de } \mathrm{HDL} \text { (0.98-1.53 mmol/L). } \\
\text { El aceite de amaranto incremento la excreción fecal de } \\
\text { colesterol ( } 180.6-424.6 \mathrm{mmol} / 3 \text { días) y ácidos biliares } \\
\text { (79.7-170.8 mmol/3 días) y el grano sólo afectó a los } \\
\text { ácidos biliares (79.7-170.8 mmol/3 días). } \\
\text { El escualeno del amaranto mostró disminución de }\end{array}$ & $\begin{array}{l}\text { Shin et al. } \\
(2004)\end{array}$ \\
\hline
\end{tabular}


Dietas (20\% proteína, 25-30 $\%$ grasa y $50-55 \%$ carbohidratos) con $20 \mathrm{~mL}$ de aceite de uva o de amaranto por día.

Ejercicio de respiración (30 $\mathrm{min} / \mathrm{día})$, cardiovascular $(60$ min/día) y de fuerza (30 $\min /$ día).

Dieta hipercolesterolémica e hipercolesterolemica con aceite de amaranto $(8 \%)$ y otra con escualeno (4.6\%).

Análisis antropométrico y bioquímico (colesterol total HDL, LDL, TG y glucosa)

Colesterol sérico y hepático, análisis histológico y ácidos biliares.

Administración oral de escualeno $(0.01,0.05$ y 0.1 $\mathrm{mL} /$ día).

Ratas Dosis únicas de $0.1 \mathrm{~mL}$ de

Wistar escualeno oral en ratas de 95-115 g

Dosis única de escualeno

$(0.1 \mathrm{~mL})$

Ratones

deficientes Dietas enriquecidas en en Apoa-1 y escualeno $(1 \mathrm{~g} / \mathrm{kg})$.

Apoe. perfil lipídico,

excreción fecal de esteroides.

Peso corporal y hepático.

Colesterol, triglicéridos, glucosa y estrés triglicéridos (3.51-3.03 mmol/L) y colesterol total (6.08$4.72 \mathrm{mmol} / \mathrm{L})$ séricos y hepáticos $(81.6-60.27 \mathrm{mmol} / \mathrm{g}$ y 35.91-26.54 mmol/g respectivamente) así como un incrementó de colesterol HDL (0.67-0.91 $\mathrm{mmol} / \mathrm{L})$ y de la excreción fecal de colesterol (169.7-283.9 mmol/3 días) y ácidos biliares (125.4-182.3 mmol/3 días).

Disminución de peso (118.5-111.9 kg), índice de masa corporal (40.6-38.9 kg/m²), circunferencias de cintura y cadera (mayor con aceite de amaranto), masa grasa,

masa magra, masa grasa visceral y agua corporal total en ambos grupos.

Reducción de glucosa en ayuno (110.5-102 mg/dL) con aceite de amaranto.

Mejora de las concentraciones de colesterol total (193.8 $179.2 \mathrm{mg} / \mathrm{dL})$, LDL (117.6-105.3 mg/dL) y TG (143-136.5 $\mathrm{mg} / \mathrm{dL}$ ) con aceite de amaranto.

Incremento en el HDL (42-5-44.5 mg/dL)

La excreción fecal de ácidos biliares fue mayor con dietas con aceite de amaranto (1.30-2.24 $\mu \mathrm{mol} \mathrm{g}^{-1}$ heces) y escualeno (1.30-2.23 $\mu \mathrm{mol} \mathrm{g}{ }^{-1}$ heces).

Menor inflamación y grado de esteatosis con la dieta hipercolesterolémica.

Aumento del colesterol total hepático $(4.11-5.19 \mathrm{mg} / \mathrm{g})$

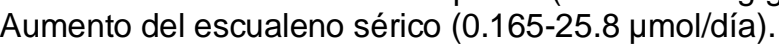

Ganancia de peso,

25 días oxidativo.
$7.79 \mu \mathrm{mol} / \mathrm{día})$

Incremento temporal en la excreción de ácidos biliares (BMCA y $\alpha \mathrm{MCA})$.

Aumento de los niveles de glucosa (20-22 mM).

Disminución en las especies reactivas de oxígeno

(EROs) de las LDL $22.5 \times 10^{5}-4 \times 10^{5}$ Unidades Arbitrarias de Fluorescencia (UAF) y aumento en la concentración plasmática de colesterol HDL (0.4-0.6 mM).

Gabás et al. Incremento de HDL en ratones salvajes (2.6-3.1 mM), sin cambio en ratones con deficiencias.
De Castro

et al. (2013)

Nakamura

et al. (1997) 
Dietas hipercolesterolémicas sustituyendo la caseína con aislado proteico (AI30) o harina de amaranto (AF30)

$(25 \%)$, y al $50 \%$ (Al7 y

AF7).
Disminución de colesterol LDL $(0.33-0.3 \mathrm{~g} / \mathrm{L})$ y

triglicéridos (1.38-1.2 g/L) en AF7, así como un

30 días (AI30 incremento de la excreción de colesterol en heces en un

y AF30) $\quad 77 \%(A F 7), 23 \%(A I 30)$ y $108 \%(A F 30)$.

7 días (Al7 y Disminución de colesterol hepático $98 \%$ (AF7), $96 \%$

AF7)

(AI30), y $53 \%$ (AF30).
Sisti et al. 


\section{Conclusión}

Debido a su composición química, la ingesta de amaranto puede aportar beneficios al perfil lipídico, a través de la fibra dietética o la fracción lipídica, la cual contiene cantidades importantes de ácidos grasos mono y poliinsaturados, así como de escualeno, el cual ha demostrado tener actividad antioxidante, importante para el control de las especies reactivas de oxígeno y el mejoramiento del perfil lipídico para el control y la prevención de las dislipidemias.

\section{Referencias}

[1]. D'Amico S, Schoenlechner R. Amaranth: Its Unique Nutritional and Health-Promoting Attributes [Internet]. Gluten-Free Ancient Grains: Cereals, Pseudocereals, and Legumes: Sustainable, Nutritious, and Health-Promoting Foods for the 21st Century. Elsevier Ltd; 2017. 131159 p. Available from: http://dx.doi.org/10.1016/B978-0-08-1008669/00006-6

[2]. Alvarez-Jubete L, Arendt EK, Gallagher E. Nutritive value of pseudocereals and their increasing use as functional gluten-free ingredients. Trends Food Sci Technol [Internet]. 2010; 21(2):106-13. Available from: http://dx.doi.org/10.1016/j.tifs.2009.10.014

[3]. Pirzadah TB, Malik B. Pseudocereals as super foods of 21st century: Recent technological interventions. J Agric Food Res [Internet]. 2020; 2(May):100052. Available
https://doi.org/10.1016/j.jafr.2020.100052

[4]. Bressani R. Amaranth. Encycl Food Sci Nutr. 2003; 52(2):2525-31.

[5]. Schröter D, Baldermann S, Schreiner M, Witzel K, Maul R, Rohn S, Neugart S. Natural diversity of hydroxycinnamic acid derivatives, flavonoid glycosides, carotenoids and chlorophylls in leaves of six different amaranth species. Food Chem [Internet]. 2018; 267:376-86. Available from: https://doi.org/10.1016/j.foodchem.2017.11.043

[6]. Escalante Escoffié MC. Rescate y revaloración del cultivo del amaranto. Ciudad de México: Instituto Interamericano de Cooperación para la Agricultura (IICA); 2010. 1-89 p.

[7]. Chmelík Z, Šnejdrlová M, Vrablík M. Amaranth as a potential dietary adjunct of lifestyle modification to improve cardiovascular risk profile [Internet]. Vol. 72, Nutrition Research. Elsevier Inc.; 2019 [cited 2020 Aug 28]. p. 36-45. Available from: https://linkinghub.elsevier.com/retrieve/pii/S0271531719302568

[8]. Soares RA, Mendonça S, De Castro L, Menezes A, Arêas J. Major peptides from Amaranth (Amaranth cruentus) protein inhibit HMG.CoA reductase activity. Int J Mol Sci. 2015; 16:4150-60.

[9]. Vilcacundo R, Martínez-Villaluenga C, Miralles B, HernándezLedesma B. Release of multifunctional peptides from kiwicha (Amaranthus caudatus) protein under in vitro gastrointestinal digestion. J Sci Food Agric. 2019; 99(3):1225-32.

[10]. Pond W, Lehmann J, Elmore R. Feeding value of raw a heated grain amaranth germplasm. Anim Feed Sci Technol. 1991; 33(221236).
[11]. Palombini SV, Claus T, Maruyama A, Gohara AK, Henrique A, Souza P, Evelázio De Souza N, Vergílio Visentainer J, Terezinha S, Gomes M, Matsushita M. Evaluation of nutritional compounds in new amaranth and quinoa cultivars. Food Sci Technol, Campinas [Internet]. [cited 2020 Aug 24];33(2):339-44. Available from: http://dx.doi.org/10.1590/S0101-20612013005000051

[12]. Gorinstein S, Pawelzik E, Delgado-Licon E, Haruenkit R, Weisz $\mathrm{M}$, Trakhtenberg S. Characterisation of pseudocereal and cereal proteins by protein and amino acid analyses. J Sci Food Agric. 2002; 82(8):886-91.

[13]. Gorinstein S, Jaramillo NO, Medina OJ, Rogriques WA, Tosello GA, Paredes-Lopez O. Evaluation of some cereals, plants and tubers through protein composition. J Protein Chem. 1999;18(6):687-93.

[14]. Nieto C. El cultivo de amaranto (Amaranthus spp) una alternativa agronómica para Ecuador. INIAP. 1990; 52.

[15]. Januszewska-Jóźwiak K, Synowiecki J. Characteristics and suitability of amaranth components in food biotechnology. Biotechnologia. 2008; 3:89-102.

[16]. Silva-Sánchez C, González-Castañeda J, de León-Rodríguez A, Barba de la Rosa A. Functional and rheological properties of amaranth albumins extracted from two Mexican varieties. Plant Foods Hum Nutr. $2004 ; 4: 169-174$

[17]. Tosi EA, Lucero H, Masciarelli R. Dietary fiber obtained from amaranth (Amaranthus cruentus) grain by differential milling. Food Chem. 2001; 73:441-3.

[18.] Wolosik K, Markowska A. Amaranthus cruentus taxonomy, botanical description, and review of its seed chemical composition. Nat Prod Commun. 2019; 14(5).

[19]. Mustafa AF, Seguin P, Gélinas B. Chemical composition, dietary fibre, tannins and minerals of grain amaranth genotypes. Int $\mathbf{J}$ Food Sci Nutr. 2011;62(7):750-4.

[20]. Badui Dergal S. Quimica de los alimentos. Cuarta edi. Pearson Educación; 2006.

[21]. Tang Y, Tsao R. Phytochemicals in quinoa and amaranth grains and their antioxidant, anti-inflammatory, and potential health beneficial effects: a review. Mol Nutr Food Res. 2017; 61(7):1-16.

[22]. Luis GM, Rebeca B, Hernández H, Peña V, Guillermo N, López T, Adrián V, Martínez E, Pacheco R. Current and potential uses of Amaranth (Amaranthus spp.). J Negat No Posit Results. 2018; 3(6):423-36.

[23]. Cai YZ, Corke H, Wu HX. Amaranth. Encycl Grain Sci. 2004; $1-10$

[24]. Martinez-Lopez A, Millan-Linares MC, Rodriguez-Martin NM, Millan F, Montserrat-de la Paz S. Nutraceutical value of kiwicha (Amaranthus caudatus L.). J Funct Foods [Internet]. 2020; 65(December):103735. Available from: https://doi.org/10.1016/j.jff.2019.103735

[25]. Özer NK, Azzi A. Effect of vitamin E on the development of nutritional hypercholesterinemia and atherosclerosis. Monatsschr Unfallheilkd Versicherungsmed. 1960; 6:62-72.

[26]. Bruni R, Medici A, Scalia S, Poli F, Muzzoli M, Sacchetti G. Wild Amaranthus cauda Seed Oil, a Nutraceutical Resource from. J Agric Food Chem. 2001; 49:5455-60. 
[28]. Kraujalis P, Venskutonis PR, Pukalskas A, Kazernavi R. LWT Food Science and Technology Accelerated solvent extraction of lipids from Amaranthus spp . seeds and characterization of their composition. $2013 ; 54: 528-34$.

[29]. Fidalgo Rodríguez JL, Dynarowicz-Latka P, Miñones Conde J. How unsaturated fatty acids and plant stanols affect sterols plasma level and cellular membranes? Review on model studies involving the Langmuir monolayer technique. Chem Phys Lipids. 2020; 232(September)

[30]. Domínguez-Avila AA, González-Aguilar GA. Lipids. In: Postharvest Physiology and Biochemistry of Fruits and Vegetables [Internet]. Elsevier; 2018 [cited 2020 Aug 24]. p. 273-92. Available from:

https://linkinghub.elsevier.com/retrieve/pii/B9780128132784000130

[31]. FAO, OMS. Norma para aceites vegetales especificados CXS 210 - 1999. Códex Aliment Normas Int los Aliment [Internet]. 2019; Available from: http://www.fao.org/fao-who-codexalimentarius/shproxy/en/? lnk=1\&url=https $\% 253 \mathrm{~A} \% 252 \mathrm{~F} \% 252 \mathrm{Fworkspace}$.fao.org\% 252Fsites $\% 252 \mathrm{Fcodex} \% 252 \mathrm{FStandards} \% 252 \mathrm{FCXS} \% 2 \mathrm{~B} 210$ $1999 \% 252 F C X S \_210$ s.pdf

[32]. Popa O, Bəbeanu NE, Popa I, Niţə S, Dinu-Pârvu CE. Methods for obtaining and determination of squalene from natural sources. Biomed Res Int. 2015;2015.

[33]. FAO. Capítulo 5 - Elaboración y refinado de aceites comestibles [Internet]. [cited 2020 Oct 17]. Available from: http://www.fao.org/3/v4700s/v4700s09.htm

[34]. Reddy LH, Couvreur P. Squalene: A natural triterpene for use in disease management and therapy. Adv Drug Deliv Rev [Internet] 2009; 61(15):1412-26. Available from http://dx.doi.org/10.1016/j.addr.2009.09.005

[35]. Picardo M, Ottaviani M, Camera E, Mastrofrancesco A Sebaceous gland lipids. Dermatoendocrinol [Internet]. 2009;1(2):6871. Available from: http://dx.doi.org/10.4161/derm.1.2.8472

[36]. Liu GCK, Ahrens EH, Schreibman PH, Crouse JR Measurement of squalene in human tissues and plasma: validation and application. J Lipid Res. 1976; 17:38-45.

[37]. Bakes MJ, Nichols PD. Lipid, fatty acid and squalene composition of liver oil from six species of deep-sea sharks collected in southern australian waters. Comp Biochem Physiol -- Part B Biochem. 1995; 110(1):267-75.

[38]. Treberg JR, Speers-Roesch B. Does the physiology of chondrichthyan fishes constrain their distribution in the deep sea? J Exp Biol. 2016;219(5):615-25.

[39]. Lopez S, Bermudez B, Montserrat-De La Paz S, Jaramillo S, Varela LM, Ortega-Gomez A, Abia R, Muriana FJG. Membrane composition and dynamics: A target of bioactive virgin olive oil constituents. Biochim Biophys Acta - Biomembr [Internet]. 2014; 1838(6):1638-56 Available from: http://dx.doi.org/10.1016/j.bbamem.2014.01.007

[40]. Newmark HL. Squalene, olive oil, and cancer risk. Review and hypothesis. Ann N Y Acad Sci. 1999; 889:193-203.

[41]. Czaplicki S, Ogrodowska D, Derewiaka D, Tan`ska M, Tan`ska $\mathrm{T}$, Zadernowski R. Bioactive compounds in unsaponifiable fraction of oils from unconventional sources. 2009; 61:1412-26.
[42]. Rhee E, Kim HC, Kim JH, Lee EY, Kim BJ, Kim EM, Song Y, Lim JH, Kim HJ, Choi S, Moon MK, Na JO, Park K, Oh MS, Han SY, Noh J, Yi KH, Lee S, Hong S, Jeong I. 2018 Guidelines for the management of dyslipidemia Cardiovascular disease in Koreans. Korean J Intern Med [Internet]. 2019; 34(4):723-71. Available from: http://kjim.org/journal/view.php?doi=10.3904/kjim.2019.188

[43]. Pisoschi AM, Pop A. The role of antioxidants in the chemistry of oxidative stress: A review. Eur J Med Chem [Internet]. 2015; 97:5574. Available from: http://dx.doi.org/10.1016/j.ejmech.2015.04.040

[44]. Kelly GS. Squalene and its potential clinical uses. Altern Med Rev. 1999; 4(1):29-36.

[45]. Bruce KD, Byrne CD. The metabolic syndrome: Common origins of a multifactorial disorder. Postgrad Med J. 2009;85(1009):614-21.

[46]. NORMA Oficial Mexicana NOM-037-SSA2-2002, Para la prevención, tratamiento y control de las dislipidemias [Internet]. 2002 [cited 2019 Oct 27]. Available from: http://www.salud.gob.mx/unidades/cdi/nom/037ssa202.html

[47]. OMS. OPS/OMS | Enfermedades crónicas, el peor asesino [Internet]. [cited 2020 Jun 23]. Available from: https://www.paho.org/hq/index.php?option=com content\&view=artic le\&id=7656:2010-enfermedades-cronicas-peorasesino $\&$ Itemid $=4327 \&$ lang $=$ es

[48]. Meaney E, Vela A, Ramos A, Alemao E, Yin D. Cumplimiento de las metas con reductores del colesterol en pacientes mexicanos. El estudio COMETA México. Gac Med Mex. 2004;140(5):493-501.

[49]. Secretaria de salud. Diagnóstico y tratamiento de dislipidemias (Hipercolesterolemia) en el adulto. Encyclopedia of Conscientiology. 2016. 1-68 p

[50]. Qureshi A, Lehmann J, Peterson D. Amaranth and Its Oil Inhibit Cholesterol Biosynthesis in 6 week old female chickens. World. 1996 ;(November 1995):1972-8.

[51]. Berger A, Gremaud G, Baumgartner M, Rein D, Monnard I, Kratky E, Geiger W, Burri J, Dionisi F, Allan M, Lambelet P. Cholesterol-lowering properties of Amaranth grain and oil in hamsters. Int J Vitam Nutr Res. 2003;73(1):39-47.

[52]. De Castro LÍA, Soares RAM, Saldiva PHN, Ferrari RA, Miguel AMRO, Almeida CAS, Arêas JAG. Amaranth oil increased fecal excretion of bile acid but had no effect in reducing plasma cholesterol in hamsters. Lipids. 2013; 48(6):609-18

[53]. Caselato-Sousa VM, Ozaki MR, De Almeida EA, AmayaFarfan J. Intake of heat-expanded amaranth grain reverses endothelial dysfunction in hypercholesterolemic rabbits. Food Funct. 2014; 5(12):3281-6

[54]. Moszak M, Zawada A, Juchacz A, Grzymislawski M, Bogdanski P. Comparison of the effect of rapeseed oil or amaranth seed oil supplementation on weight loss, body composition, and changes in the metabolic profile of obese patients following 3-week body mass reduction program: a randomized clinical trial. Lipids Health Dis. $2020 ; 19$.

[55]. Nakamura Y, Tonogai Y, Tsumura Y, Shibata T, Uchiyama M. Effect of dietary squalene on the fecal steroid excretions and the lipid levels of serum and the liver in the rat. Nutr Res. 1997; 17(2):243-2577. 
56. Shin DH, Heo HJ, Lee YJ, Kim HK. Amaranth squalene reduces serum and liver lipid levels in rats fed a cholesterol diet. Br J Biomed Sci. 2004; 61(1):11-4.

[57]. Gabás-Rivera C, Barranquero C, Martínez-Beamonte R, Navarro MA, Surra JC, Osada J. Dietary squalene increases high density lipoprotein-cholesterol and paraoxonase 1 and decreases oxidative stress in mice. PLoS One. 2014; 9(8)

[58]. Sisti MS, Scilingo A, Añón MC. Effect of the Incorporation of Amaranth (Amaranthus Mantegazzianus) into Fat- and CholesterolRich Diets for Wistar Rats. J Food Sci. 2019;84(11):3075-82.

[59]. Romero-Martínez M, Shamah-Levy T, Cuevas-Nasu L, GómezHumarán IM, Gaona-Pineda EB, Gómez-Acosta LM, RiveraDommarco JÁ, Hernández-Ávila M. Encuesta Nacional de Salud y Nutrición de Medio Camino 2016. Salud Publica Mex [Internet] 2016;59(3):299-305. Available from http://www.ncbi.nlm.nih.gov/pubmed/28902317

[60]. Instituto Nacional de Salud Pública. Encuesta Nacional de Salud y Nutrición: Presentación de resultados [Internet]. Vol. 1, Ensanut. 2018. p. 47. Available from: https://ensanut.insp.mx/encuestas/ensanut2018/doctos/informes/ensan ut_2018_presentacion_resultados.pdf

[61]. OMS. Cardiovascular diseases (CVDs) [Internet]. 2017 [cited 2021 Apr 18]. Available from: https://www.who.int/en/news room/fact-sheets/detail/cardiovascular-diseases-(cvds)

[62]. OMS. Información general sobre la hipertensión en el mundo [Internet]. Ginebra; 2013 [cited 2021 Apr 18]. Available from www.who.int

[63]. Arreola Peñalosa M, Arriega Dávila J, Pérez Rodríguez G, López Ocaña L, Viviegra Osorio A, Sepúlveda Vildosola A Diagnóstico y tratamiento de las dislipidemias (hipercolesterolemia). Vol. 67, Instituto Mexicano del Seguro Social. Ciudad de México 2016

[64]. L'homme L, Esser N, Riva L, Scheen A, Paquot N, Piette J, Legrand-Poels S. Unsaturated fatty acids prevent activation of NLRP3 inflammasome in human monocytes/macrophages. J Lipid Res. 2013 54(11):2998-3008

[65]. Micera M, Botto A, Geddo F, Antoniotti S, Bertea CM, Levi R, Gallo MP, Querio G. Squalene : More than a Step toward Sterols. 2020 $1-14$.

[66]. Hien HTM, Ha NC, Thom LT, Hong DD. Squalene promotes cholesterol homeostasis in macrophage and hepatocyte cells via activation of liver X receptor (LXR) $\alpha$ and $\beta$. Biotechnol Lett. 2017; 39(8):1101-7.

[67]. Geyeregger R, Zeyda M, Stulnig TM. Liver X receptors in cardiovascular and metabolic disease. Cell Mol Life Sci. 2006; 63(5):524-39.

[68]. Soliman GA. Dietary fiber, atherosclerosis, and cardiovascula disease. Nutrients [Internet]. 2019; 1829-43. Available from: http://dx.doi.org/10.1016/B978-1-4377-0398-6.00086-X

[69]. Cummings JH. Short chain fatty acids in the human colon [Internet]. Vol. 22, Gut. 1981 [cited 2021 Feb 17]. p. 763-79. Available from: https://gut.bmj.com/lookup/doi/10.1136/gut.22.9.763

[70]. Page MA, Tubbs PK. Some Properties of 3-Hydroxy-3 methylglutaryl-Coenzyme A Synthase from Ox Liver. Vol. 173 , Biochem. J. 1978.
[71]. Bush RS, Milligan LP. Study of the mechanisms of inhibition of ketogenesis by propionate in bovine liver. Can J Anim Sci [Internet]. 1971 Apr 1 [cited 2021 Feb 17]; 51(1):121-7. Available from: http://www.nrcresearchpress.com/doi/10.4141/cjas71-016

[72]. Gil G, Hegardt FG. Some properties of purified 3-hydroxy-3methylglutaryl coenzyme A reductase phosphatases from rat liver. Arch Biochem Biophys [Internet]. 1982 Mar [cited 2021 Feb 17]; 214(1):192-8. Available from: https://linkinghub.elsevier.com/retrieve/pii/0003986182900224

[73]. Yegin S, Kopec A, Kitts DD, Zawistowski J. Dietary fiber: a functional food ingredient with physiological benefits. In: Dietary Sugar, Salt and Fat in Human Health [Internet]. Elsevier; 2020 [cited 2021 Feb 19]. p. 531-55. Available from: https://linkinghub.elsevier.com/retrieve/pii/B978012816918600024X

[74]. Gallaher DD, Hassel CA, Lee KJ, Gallaher CM. Viscosity and fermentability as attributes of dietary fiber responsible for the hypocholesterolemic effect in hamsters. J Nutr [Internet]. 1993 [cited 2021 Feb 18]; 123(2):244-52. Available from: https://pubmed.ncbi.nlm.nih.gov/8381479/

[75]. Reppas C, Swidan SZ, Tobey SW, Turowski M, Dressman JB. Hydroxypropylmethylcellulose significantly lowers blood cholesterol in mildly hypercholesterolemic human subjects. Eur J Clin Nutr. 2009; 63(1):71-7.

[76]. Dongowski G. Interactions between dietary fibre-rich preparations and glycoconjugated bile acids in vitro. Food Chem. 2007 Jan 1; 104(1):390-7.

[77]. Cornfine C, Hasenkopf K, Eisner P, Schweiggert U. Influence of chemical and physical modification on the bile acid binding capacity of dietary fibre from lupins (Lupinus angustifolius L.). Food Chem [Internet]. 2010 Oct 1 [cited 2021 Feb 18]; 122(3):638-44. Available from:

https://linkinghub.elsevier.com/retrieve/pii/S0308814610002840

[78]. Zacherl C, Eisner P, Engel KH. In vitro model to correlate viscosity and bile acid-binding capacity of digested water-soluble and insoluble dietary fibres. Food Chem [Internet]. 2011 May 15 [cited 2021 Feb 18]; 126(2):423-8. Available from: https://linkinghub.elsevier.com/retrieve/pii/S0308814610014068

[79]. Ghaffarzadegan T, Zhong Y, Fåk Hållenius F, Nyman M. Effects of barley variety, dietary fiber and $\beta$-glucan content on bile acid composition in cecum of rats fed low- and high-fat diets. J Nutr Biochem [Internet]. 2018 Mar 1 [cited 2021 Feb 18]; 53:104-10. Available from: https://linkinghub.elsevier.com/retrieve/pii/S0955286317304400

[80]. Xue Z, Gao X, Jia Y, Wang Y, Lu Y, Zhang M, Panichayupakaranant $\mathrm{P}$, Chen $\mathrm{H}$. Structure characterization of high molecular weight soluble dietary fiber from mushroom Lentinula edodes (Berk.) Pegler and its interaction mechanism with pancreatic lipase and bile salts. Int J Biol Macromol. 2020 Jun 15; 153:1281-90. T, Valladares A, Lizán L, Sacristán JA. Adherencia y persistencia terapéutica: causas, consecuencias y estrategias de mejora. Aten. Primaria 2009; 41(6): 342-48. 